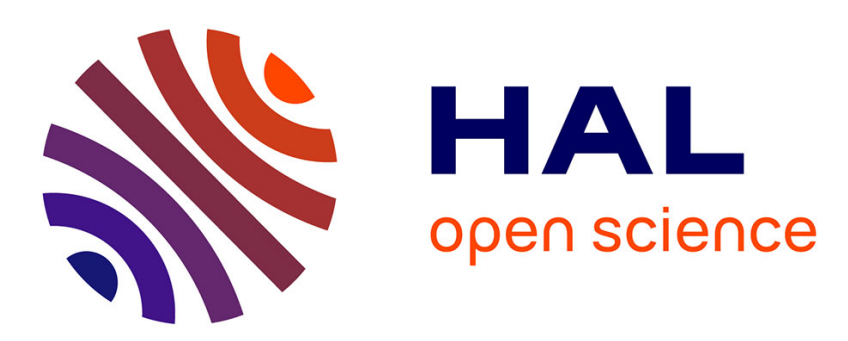

\title{
Simulation de la cinétique des transitions électroniques entre les niveaux énergétiques d'un semiconducteur doté d'impuretés
}

J. Brini, G. Kamarinos, P. Viktorovitch

\section{- To cite this version:}

J. Brini, G. Kamarinos, P. Viktorovitch. Simulation de la cinétique des transitions électroniques entre les niveaux énergétiques d'un semiconducteur doté d'impuretés. Revue de Physique Appliquée, 1974, 9 (2), pp.451-454. 10.1051/rphysap:0197400902045100 . jpa-00243801

\section{HAL Id: jpa-00243801 https://hal.science/jpa-00243801}

Submitted on 1 Jan 1974

HAL is a multi-disciplinary open access archive for the deposit and dissemination of scientific research documents, whether they are published or not. The documents may come from teaching and research institutions in France or abroad, or from public or private research centers.
L'archive ouverte pluridisciplinaire HAL, est destinée au dépôt et à la diffusion de documents scientifiques de niveau recherche, publiés ou non, émanant des établissements d'enseignement et de recherche français ou étrangers, des laboratoires publics ou privés. 


\title{
SIMULATION DE LA CINÉTIQUE DES TRANSITIONS ÉLECTRONIQUES ENTRE LES NIVEAUX ÉNERGÉTIQUES D'UN SEMICONDUCTEUR DOTÉ D'IMPURETÉS
}

\author{
J. BRINI, G. KAMARINOS et P. VIKTOROVITCH \\ Ecole Nationale Supérieure d'Electronique et de Radioélectricité, \\ 23, rue des Martyrs, 38031 Grenoble-Cedex, France
}

(Reçu le 16 août 1973)

\begin{abstract}
Résumé. - Nous simulons la cinétique des interactions entre électrons libres, trous libres et impuretés dans un semiconducteur dont l'état stationnaire peut être hors de l'équilibre thermodynamique. L'identité formelle des équations de Shockley-Read avec des équations de cinétique chimique nous permet d'utiliser un calculateur original destiné à simuler des réseaux de réactions chimiques (en neurobiologie par exemple). Nous obtenons la confirmation en régime dynamique de résultats, déduits d'expériences de photoconductivité, concernant les propriétés de piégeage des niveaux d'impuretés. La simulation nous conduit à dégager une méthode permettant de déterminer les caractéristiques des impuretés (niveau énergétique, densité, coefficients d'émission et de captation des électrons et des trous). Nous mettons également en évidence la possibilité de prévoir le comportement de milieux dotés de plusieurs niveaux d'impuretés.
\end{abstract}

\begin{abstract}
We simulate the kinetics of interactions between free electrons, free holes and impurities in a semiconductor not necessarily in thermal equilibrium. The formal identity between Shockley-Read's and the chemical kinetic reaction equations allows us to use a special purpose computer originally designed to simulate systems of chemical reactions (for instance in neurobiology). We confirm the trapping properties of deep level impurities which were first deduced from decaying photoconductivity experiments. We evolve a method of determining the characteristics of impurities (energy level, density, probability of emission and capture of electrons and holes). We also show the feasibility of predicting the behaviour of semiconductors containing several impurities.
\end{abstract}

1. Introduction. - Les impuretés ou défauts introduisant des niveaux énergétiques localisés dans la bande interdite d'un semiconducteur jouent un rôle fondamental dans les processus de recombinaison des électrons et des trous [1]. En particulier, la présence d'impuretés à niveaux profonds et entraînant des durées de vie différentes pour les électrons et les trous peut conduire à l'apparition de phénomènes de résistance négative lors de l'injection de porteurs dans le milieu [2], [3], [4]. Ces effets constituent, à l'heure actuelle, l'un des principaux points de recherche sur les phénomènes d'injection en raison de l'importance des dispositifs à relaxation dans les systèmes électroniques. Il est donc d'un intérêt primordial de déterminer les caractéristiques de ces impuretés (niveaux énergétiques, densité, coefficients de captation et d'émission des électrons et des trous).

A cet effet, nous simulons sur un calculateur la cinétique des interactions entre électrons libres (de densité $n$ ), trous libres (de densité $p$ ) et impuretés (de densité $N_{\mathrm{r}}$ ), dans un semiconducteur.
Nous admettons que cette cinétique est décrite par les équations de Shockley-Read.

Si les impuretés introduisent un niveau unique $E_{\mathrm{r}}$, on peut écrire :

$$
\left\{\begin{aligned}
\mathrm{d} n / \mathrm{d} t & =-C_{n} p_{\mathrm{r}} n+\gamma_{n} n_{\mathrm{r}}+g_{n} \\
\mathrm{~d} p / \mathrm{d} t & =-C_{p} n_{\mathrm{r}} p+\gamma_{p} p_{\mathrm{r}}+g_{p} \\
n_{\mathrm{r}} & =p-n \\
N_{\mathrm{r}} & =n_{\mathrm{r}}+p_{\mathrm{r}}
\end{aligned}\right.
$$

$g_{n}$ et $g_{p}$ sont les taux de création (ou de disparition) des électrons et des trous libres. Les notations utilisées sont celles de Baron et Mayer [5].

Nous utilisons un calculateur original [6] réalisé dans le laboratoire en vue de résoudre des problèmes de cinétique chimique en neurobiologie. En effet, on peut démontrer que les équations de Shockley-Read s'identifient à des équations de cinétique chimique [7]. Le principe du calculateur repose sur l'analogie « bitunité de matière » dans le cas de la biologie, sur l'ana- 
logie « bit-collection de particules » dans le cas étudié.

Les unités ainsi définies sont accumulées dans des compteurs-décompteurs jouant le rôle de sommateursintégrateurs et transférées à l'aide de multiplieurs de taux jouant le rôle de multiplicateurs.

Le calculateur ainsi réalisé est l'équivalent d'une machine analogique dont la définition serait d'undix millième de la grandeur maximale affichable. Par conséquent, les variables à traiter ne doivent pas dépasser une dynamique de $10^{4}$ et la précision maximale est de $10^{-4}$. De telles performances ne sont pas atteintes actuellement par les calculateurs analogiques classiques. La précision sur le temps permet d'observer l'évolution des variables unité par unité.

Le calculateur permet de visualiser aisément sur un oscilloscope l'évolution temporelle des variables grâce à un retour périodique aux conditions initiales choisies. Il est clair que, pour $t$ tendant vers l'infini, on obtient un état final qui nous donne les valeurs stationnaires des variables. Ces valeurs pourraient être obtenues par un calcul sur ordinateur.

Par contre, l'exploration du régime transitoire nécessiterait un calcul extrêmement lourd pour un ordinateur classique en raison $\mathrm{du}$ caractère non linéaire des équations.

2. Conditions de calcul et analyse des résultats. Imposant initialement un écart $\Delta n_{\mathrm{i}}=\Delta p_{\mathrm{i}}\left(\Delta n_{\mathrm{r}_{\mathrm{i}}}=0\right)$ des densités d'électrons libres et de trous libres par rapport au régime stationnaire (correspondant ou non à l'équilibre thermodynamique), nous étudions l'évolution temporelle des trois variables : $n, n_{\mathrm{r}}$ et $p$.

On appelle : $n_{0}, n_{\mathrm{r}_{0}}, p_{0}, p_{1_{0}}$, les densités à l'équilibre thermodynamique, $n_{\mathrm{s}}, n_{\mathrm{r}_{\mathrm{s}}}, p_{\mathrm{s}}, p_{\mathrm{r}_{\mathrm{s}}}$, les densités en régime stationnaire (hors de l'équilibre).

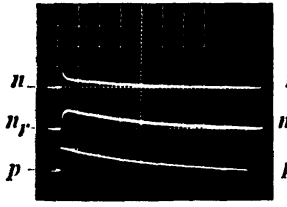

(a)

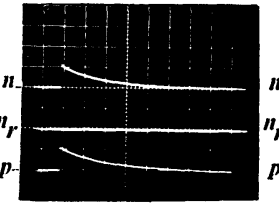

(b)

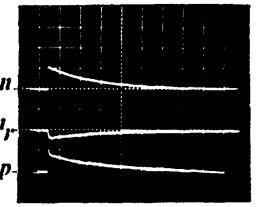

(c)
FIG. 1. - Echelles $: n$ et $p: 2 \times 10^{21} \mathrm{~m}^{-3}$ par division, $n_{\mathrm{r}}: 10^{21} \mathrm{~m}^{-3}$ par division, temps : $2 \times 10^{-6} \mathrm{~s}$ par division.

La figure 1 donne la forme des oscillogrammes typiques obtenus dans les trois cas suivants:

a) $C_{n} p_{\mathrm{r}_{0}}>C_{p} n_{\mathrm{r}_{0}}$. Dans ce cas, nous voyons que le niveau d'impureté se comporte comme un piège à électrons [4]. En effet, la décroissance de l'excès d'électrons est plus rapide que celle de l'excès de trous et le taux d'occupation en électrons du niveau d'impureté passe par un extrémum $n_{\mathrm{r}_{\mathrm{s}}}+\Delta n_{\mathrm{r}_{\mathrm{m}}}$ qui est un maximum.

b) $C_{n} p_{\mathrm{r}_{0}}<C_{p} n_{\mathrm{r}_{0}}$. Le niveau d'impureté, à l'inverse $\mathrm{du}$ cas précédent, se comporte comme un piège à trous $\left(n_{\mathrm{r}_{\mathrm{s}}}+\Delta n_{\mathrm{r}_{\mathrm{m}}}\right.$ est un minimum). c) Dans le cas où $C_{n} p_{\mathrm{r}_{0}}=C_{p} n_{\mathrm{r}_{0}}$, le niveau d'im pureté se comporte comme un centre de recombinaison idéal. Le taux d'occupation reste invariant et les décroissances des deux excès $\Delta n$ et $\Delta p$ sont identiques.

Les oscillogrammes nous ont permis d'observer que l'instant $t_{\mathrm{m}} \mathrm{du}$ passage par un extrémum du taux d'occupation du niveau d'impureté correspond à une rupture de pente des deux courbes d'évolution de $n$ et $p$. C'est cette rupture de pente qu'on observe expérimentalement lors de la décroissance de la photoconductivité d'un barreau de semiconducteur après une brève illumination [1], [4]. Nous allons plus particulièrement nous intéresser aux variations de $\Delta n_{\mathrm{r}_{\mathrm{m}}}$ en fonction du rapport $C_{p} / C_{n}$ et de $g_{n}$ et $g_{p}$.

2.1 CAS OU LE RÉGIME STATIONNAIRE CORRESPOND A L'ÉQUILIBRE THERMODYNAMIQUE $\left(g_{n}=g_{p}=0\right)$. - Sur la figure 2, nous avons tracé, pour une valeur donnée de $\Delta n_{\mathrm{i}}$, les variations de $\Delta n_{\mathrm{r}_{\mathrm{m}}}$ en fonction de $C_{p} / C_{n}$. On constate bien que, pour la valeur correspondant à $C_{p} n_{\mathrm{r}_{0}}=C_{n} p_{\mathrm{r}_{0}}, \Delta n_{\mathrm{r}_{\mathrm{m}}}$ change de signe. Dans les cas limites où $C_{p}$ ou $C_{n}$ tend vers $0\left(C_{n}\right.$ ou $C_{p}$ tend vers l'infini), la limite de $\Delta n_{r_{m}}$ est définie par $\Delta n_{\mathrm{i}}$ et l'une des deux équations cinétiques qui se réduit à une pseudo-loi d'action de masse entre le niveau d'impureté et la bande de valence ou la bande de conduction. Physiquement, ces cas correspondent à l'impossibilité de la recombinaison par le biais de ce centre des paires injectées initialement.

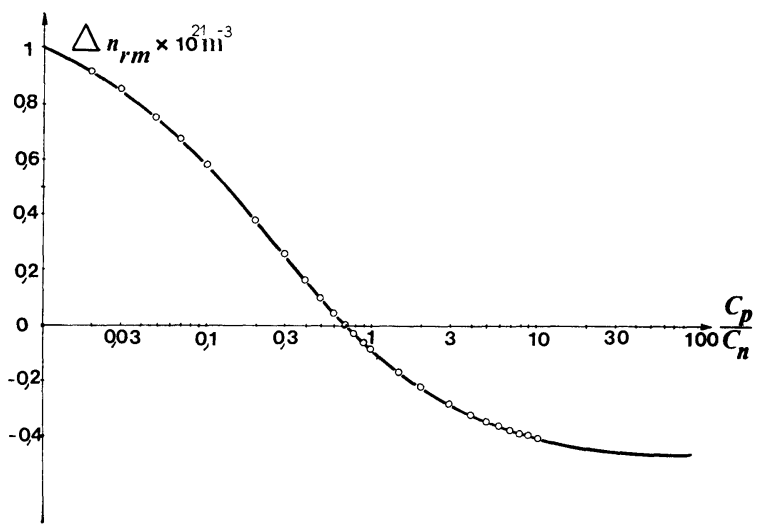

FIG. 2.

Remarques. - 1) Le rapport $C_{p} / C_{n}$ est bien le paramètre unique caractérisant la cinétique du phénomène ; en effet, lorsque $g_{n}=g_{p}=0$, multiplier $C_{n}$ et $C_{p}$ par une même constante, revient à dilater l'échelle des temps.

2) Pour une valeur donnée du rapport $C_{p} / C_{n}$, $\Delta n_{\mathrm{r}_{\mathrm{m}}}$ est une fonction monotone de $\Delta n_{\mathrm{i}}$ et tend, pour des valeurs très grandes et positives de $\Delta n_{\mathbf{i}}$, vers une limite finie correspondant à $C_{p} n_{\mathrm{r}}=C_{n} p_{\mathrm{r}}$.

2.2 CAS OU L'ÉTAT STATIONNAIRE EST DISTINCT DE L'ÉQUILIBRE THERMODYNAMIQUE $\left(\begin{array}{l}g_{n} \neq 0 \\ g_{p} \neq 0\end{array}\right)$. - Dans 
ce cas, on est en présence d'une transition entretenue entre les divers niveaux considérés (bande de valence, bande de conduction, niveau d'impureté). Nous nous limiterons à la situation où $g_{n}=g_{p}=g$, ce qui correspond à une transition directe entre bande de valence et bande de conduction. Cette situation se rencontre chaque fois qu'en régime stationnaire le semiconducteur est le siège de courants de diffusion d'électrons et de trous.

Les figures 3 et 4 montrent que les propriétés de piégeage des centres sont accrues pour des valeurs négatives de $g$ et réduites au contraire pour des valeurs positives de $g$.

La figure 3 représentant l'évolution de $n_{\mathrm{r}_{\mathrm{s}}}$ en fonction de $g$ pour diverses valeurs de $C_{p} / C_{n}$ montre en effet que les quantités $C_{p} n_{\mathrm{r}_{\mathrm{s}}}$ et $C_{n} p_{\mathrm{r}_{\mathrm{s}}}$ divergent l'une de l'autre pour $g<0$. Pour $g>0$ et très grand, les valeurs limites sont telles que, dans tous les cas, on a : $C_{p} n_{\mathrm{r}:}=C_{n} p_{\mathrm{rs}_{\mathrm{s}}}$.

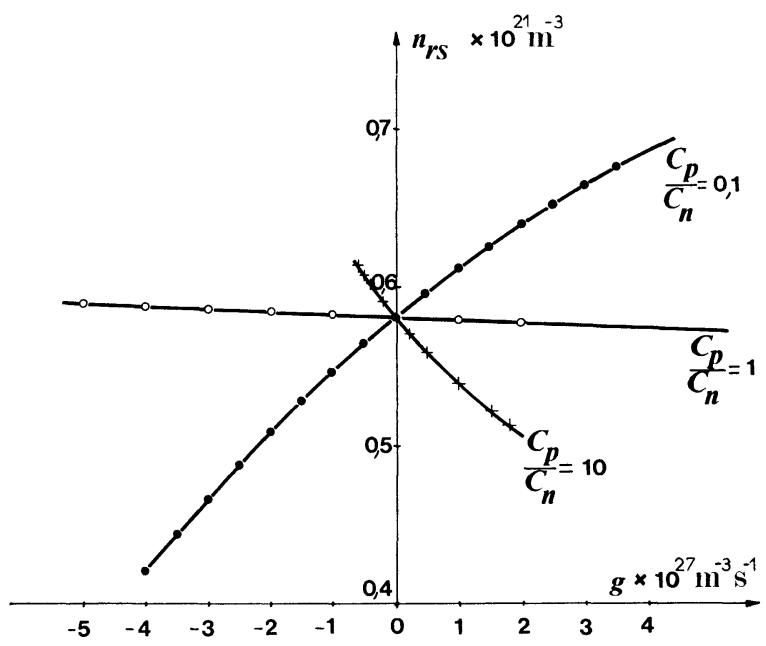

FIG. 3.

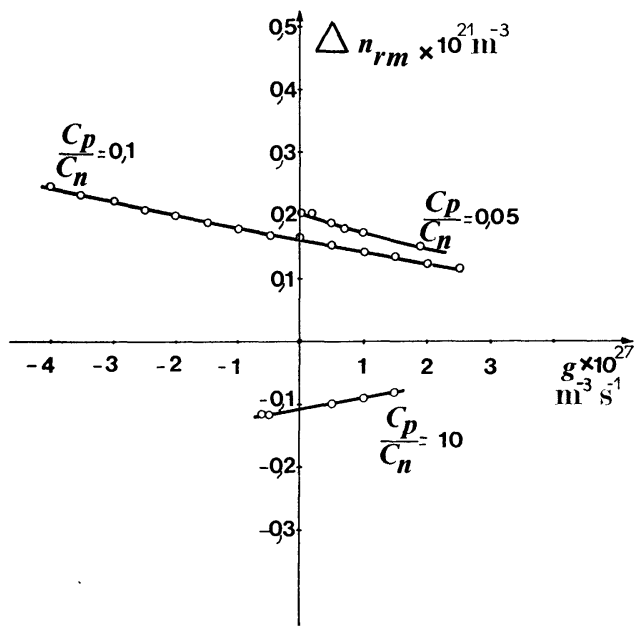

FIG. 4.
La figure 4 confirme, en régime dynamique, les résultats obtenus en régime stationnaire: en effet, $\Delta n_{\mathrm{r}_{\mathrm{m}}}$ tend vers 0 pour $g>0$ et croît en valeur absolue pour $g<0$. Pour $C_{p} n_{\mathrm{r}_{0}}=C_{n} p_{\mathrm{r}_{0}}$, on a $\Delta n_{\mathrm{r}_{\mathrm{m}}}=0$ pour tout $g$.

Remarque. - Pour $g$ non nul, $C_{p} / C_{n}$ n'est plus un paramètre unique. Les trois grandeurs $g, C_{n}$ et $C_{p}$ sont définies à une constante multiplicative près. Nous avons choisi de maintenir $C_{p}$ constant et égal à $10^{-15} \mathrm{~m}^{3} \mathrm{~s}^{-1}$

3. Conclusion. - Les résultats de la simulation sont en bon accord avec les expériences de photoconductivité effectuées sur des barreaux de germanium sièges du phénomène de magnétoconcentration [4], [8]. La magnétoconcentration provoque un appauvrissement stationnaire en porteurs libres dans le volume du semiconducteur $(g<0)$.

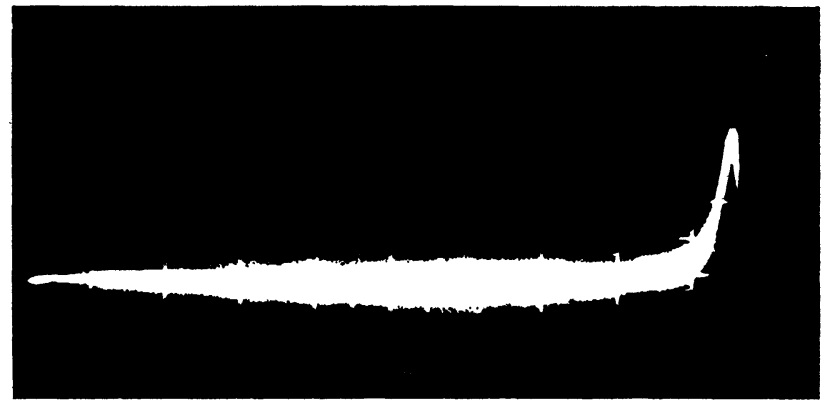

Fig. 5. - Mise en évidence d'une rupture de pente dans la décroissance de photoconductivité. Le barreau de germanium utilisé $(\rho \sim 50 \Omega . \mathrm{cm}$ à $300 \mathrm{~K})$ est doté de centres accepteurs profonds $\left(N_{\mathrm{r}} \simeq 10^{21} \mathrm{~m}^{-3}, E_{\mathrm{r}}-E_{\mathrm{v}} \simeq 0,48 \mathrm{eV}\right)$. Pendant l'expérience [4], [8], il était le siège de l'effet de magnétoconcentration $\left(B_{z}=0,8 \mathrm{~T}, E_{x}=12 \mathrm{~V} / \mathrm{cm}, T=300 \mathrm{~K}\right)$. Echelles : $200 \mathrm{mV} /$ div., $50 \mu \mathrm{s} /$ div.

Nous avons observé la décroissance de la photoconductivité du milieu après une brève illumination de ce dernier. Cette décroissance présente une rupture de pente d'autant plus importante que l'appauvrissement est plus intense. Ce comportement s'interprète aisément à la lumière des résultats de notre simulation. Celle-ci montre en effet :

- que $\left|\Delta n_{\mathrm{r}_{\mathrm{m}}}\right|$ est fonction croissante de l'appauvrissement (voir Fig. 4),

- que la rupture de pente est d'autant plus importante que $\Delta n_{\mathrm{r}_{\mathrm{m}}}$ est plus grand.

4. Perspectives. - Nous nous proposons de déterminer les caractéristiques des centres d'impuretés en confrontant les résultats expérimentaux avec ceux de la simulation :

- Dans un premier temps, des mesures de conductivité et d'effet Hall donnent les variations de $n_{0}$ et $p_{0}$ en fonction de la température (après un choix judicieux des lois des mobilités). Le calculateur 
permet alors, par approches successives, de déterminer $N_{\mathrm{r}}$ et $E_{\mathrm{r}}$ de manière à obtenir les mêmes lois de variation de $n_{0}$ et $p_{0}$ en fonction de la température.

- Confrontant ensuite les expériences de photoconductivité et la simulation en régime dynamique, on peut remonter aux coefficients cinétiques $C_{n}$ et $C_{p}$ réels.

Il est bien connu que la simulation ne se borne pas seulement à la vérification d'un modèle ou à la confrontation avec l'expérience réelle, mais qu'elle peut également s'avérer indispensable à la prévision $\mathrm{du}$ comportement de systèmes plus complexes. C'est pourquoi nous nous proposons de généraliser la simulation au cas de plusieurs niveaux d'impuretés : nous pourrons ainsi non seulement confirmer l'existence de plusieurs ruptures de pente dans l'évolution temporelle de $n$ et de $p$ (que nous avons mis en évidence lors d'expériences de photoconductivité), mais encore prévoir d'autres comportements plus complexes (par exemple : photoconductivité négative).

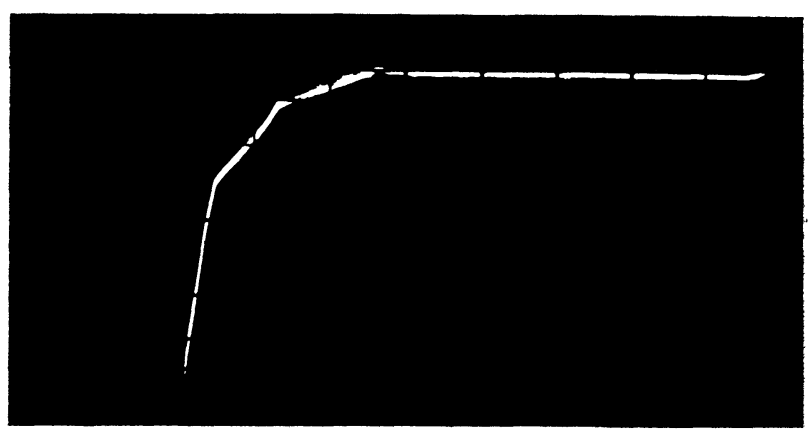

Fig. 6. - Mise en évidence de trois ruptures de pente dans la décroissance de photoconductivité sur un barreau de germanium de haute résistivité en régime de magnétoconcentration $\left(E_{x}=10 \mathrm{~V} / \mathrm{cm}, B_{z}=0,8 \mathrm{~T}, T=270 \mathrm{~K}\right)$. Echelles $: 200 \mathrm{mV} /$ div., $100 \mu \mathrm{s} / \mathrm{div}$.

\section{Bibliographie}

[1] Vapallle, A., Physique des dispositifs à semiconducteurs 1 (Masson \& Cie ed.) 1970.

[2] Ashley, K. L., Milnes, A. G., J. Appl. Phys. 35 (1964) 369.

[3] Mayer, J. W., Baron, R., Marsh, O. J., Appl. Phys. Lett. 6 (1965) 38.

[4] Viktorovitch, P., Kamarinos, G., Phys. Stat. Sol. (a) 16 (1973) 219.
[5] Baron, R., Mayer, J. W., Semiconductors and semimetals Vol. 6 : Injection Phenomena, Chap. 4, Willardson \& Beer (Acad. Press ed.) 1970.

[6] Brini, J., Herault, J., C. R. Hebd. Séan. Acad. Sci. (Paris) D 275 (1972) 2167.

[7] Viktorovitch, P., Kamarinos, G., C. R. Hebd. Séan. Acad. Sci. (Paris) $277 B$ (1973) 305.

[8] Chovet, A., Kamarinos, G., Revue Phys. Appl. 6 (1971) 345. 